\title{
The gyrotron - a natural source of high-power orbital angular momentum millimeter-wave beams
}

\author{
M. Thumm $^{1}$, A. Sawant ${ }^{2}$, M.S. Choe ${ }^{3}$, E.M. Choi ${ }^{2,3}$ \\ ${ }^{1}$ Karlsruhe Institute of Technology (KIT), IHM and IHE, Karlsruhe, Germany, manfred.thumm@kit.edu \\ ${ }^{2}$ Department of Electrical Eng., Ulsan National Inst. of Science and Technology (UNIST), Ulsan, South Korea ${ }^{3}$ Department of Phys- \\ ics, Ulsan National Institute of Science and Technology (UNIST), Ulsan, South Korea
}

\begin{abstract}
Orbital angular momentum (OAM) of electromagnetic-wave beams provides further diversity to multiplexing in wireless communication. The present report shows that higher-order mode gyrotrons are natural sources of highpower OAM millimeter ( $\mathrm{mm})$ wave beams. The welldefined OAM of their rotating cavity modes operating at near cutoff frequency has been derived by photonic and electromagnetic wave approaches.
\end{abstract}

\section{Introduction}

Orbital Angular Momentum (OAM) [1] carried by light beams (vortex beams) was discovered earlier and has been widely employed in many applications such as optical tweezers, optical drives of micro-machines, atom trapping, and optical communication. OAM provides an additional dimension (diversity) to multiplexing techniques which can be employed in addition to conventional multiplexing methods to achieve higher data rates. OAM beams have been thoroughly studied and used in the optical regime [2] but in the mm-wave region, they are still under investigation. In this frequency band, there are difficulties associated with beam-splitting and beamcombining processes as well as with the use of spiral phase plates for generation of OAM, since the wavelength is much larger compared to those at optical frequencies.

Here we introduce the natural generation of highpower OAM modes excited by gyrotrons with axial output of the rotating higher-order transverse electric mode $T E_{m, p}$ where $m$ and $p$ are the azimuthal and radial mode index, respectively. Such output radiation could be used for long-range wireless communication. To achieve high frequency stability and a narrow line width, a phase locking system can be utilized to control the electron beam energy. The long-term stability can be guaranteed by a reference clock. A line width as narrow as $1 \mathrm{~Hz}$ has been obtained [3].

\section{OAM of Rotating Gyrotron Modes}

Helically propagating rotating $T E_{m, p}$ modes in a circular waveguide can be decomposed into a series of cylindrical plane waves, each propagating at the Brillouin angle $\theta_{B}$ relative to the waveguide axis. The angular momentum possessed by such a ray (which is tangential to the caustic of the mode) can be expressed as

$$
\vec{L}=L \hat{z}=\vec{r} \times \vec{p}=R_{c} \hat{r} \times p_{\Phi} \hat{\Phi}=R_{c} p_{\Phi} \hat{z}
$$

here $R_{\mathrm{c}}=\left(m / X_{m, p}\right) R_{0}$ is the caustic radius of the operating mode, where $X_{m, p}$ is the $p^{\text {th }}$ root of the derivative of the
Bessel function $J_{m}, R_{0}$ is the waveguide radius and where $p_{\Phi}=p \sin \theta_{B}=\hbar k \sin \theta_{B}, \sin \theta_{B}=\frac{k_{\perp}}{k}$ and $k_{\perp}=\frac{\chi_{m, p}}{R_{0}}$.

Therefore, $\vec{L}=R_{c} p_{\Phi} \hat{z}=\frac{m}{\chi_{m, p}} R_{0} \hbar k \frac{\chi_{m, p}}{k R_{0}}=m \hbar \hat{z}$

The ratio between the total angular momentum $L_{N}$ and total energy $W_{N}$ of $N$ photons is given by

$$
\frac{\vec{L}_{N}}{W_{N}}=\frac{N m \hbar}{N \hbar \omega}=\frac{m}{\omega}
$$

where $\omega$ is the angular frequency of the operating cavity mode which is close to the cutoff frequency $\omega_{\text {cut }}=c X_{m, p} / R_{0}$ in the cylindrical cavity section. Here $c$ is the velocity of light in vacuum. Therefore follows $m / \omega=$ $R_{\mathrm{c}} / c$, which means that the OAM is proportional to the absolute caustic radius.

This is the result of the photonic approach to derive the OAM. The same value follows from the electromagnetic wave approach for the total OAM of a gyrotron cavity mode within a given waveguide volume per total energy of the electromagnetic wave in the same volume [4].

At a given frequency the OAM increases with increasing azimuthal mode number $m$, whereas for fixed $m$ it decreases with increasing frequency.

Modes that have a linear polarization may possess a so-called Spin Angular Momentum (SAM) if their righthand and left-hand rotation components are different. Table 1 summarizes the SAM and OAM features of different types of TE modes.

Table 1. Intensity on axis $I_{0}$, possible spin angular momentum (SAM) and orbital angular momentum (OAM) of gyrotron $T E$ modes in circular waveguides

\begin{tabular}{|c|c|c|c|}
\hline Mode Family & $\boldsymbol{I}_{\mathbf{0}}$ & $\begin{array}{c}\text { SAM } \\
\text { (Polarization) }\end{array}$ & OAM \\
\hline$T E_{0, p}$ & no & no & no \\
\hline$T E_{1, p}$ & yes & yes & no \\
\hline$T E_{m, p}(m>1)$ & no & no & yes \\
\hline
\end{tabular}

\section{Gyrotrons with OAM mm-Wave Output Beams}

UNIST has been operating a $65 \mathrm{~kW}$ W-band gyrotron with diode-type magnetron injection gun (MIG), $T E_{6,2}$ cavity mode and quasi-optical output in the linearly polarized fundamental Gaussian $T E M_{0,0}$ mode [5]. In case that the quasi-optical output mode converter would be removed and exchanged with a mode-conserving nonlinear diameter up-taper, one would have the essential feature of a OAM gyrotron with axial mm-wave output configuration where the cavity mode is directly emitted 
through the dielectric tube window into free space. Of course, propagation of higher-order OAM beams generated by gyrotrons in free space results in a large diffraction angle, which however, can be compensated by the waveguide diameter taper section that is added after the gyrotron cavity to reduce the Brillouin angle $\theta_{B}$ of the output wave and by employing focusing lenses to reduce the diffraction. Provided that the gyrotron would also be equipped with a triode-type MIG, the tube could be electronically switch between co- and counter-rotating cavity modes (right- and left hand OAM) and between two cavity modes with different magnitude of OAM (see table 2).

Table 2. Possible operating cavity modes with different OAM of the $65 \mathrm{~kW}$ UNIST W-band gyrotron

\begin{tabular}{|c|c|c|c|}
\hline $\begin{array}{c}\text { Cavity } \\
\text { Mode }\end{array}$ & $\boldsymbol{X}_{\boldsymbol{m}, \boldsymbol{n}}$ & $\begin{array}{c}\text { Frequency } \\
\text { (GHz) }\end{array}$ & $\begin{array}{c}\text { Relative Caustic } \\
\text { Radius } \boldsymbol{m} / \boldsymbol{X}_{\boldsymbol{m}, \boldsymbol{n}}\end{array}$ \\
\hline$T E_{6,2}$ & 11.734936 & 94.8 & 0.51 \\
\hline$T E_{10,1}$ & 11.770877 & 95.1 & 0.85 \\
\hline
\end{tabular}

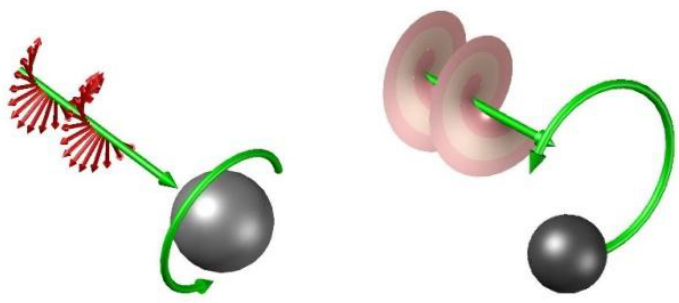

Fig. 1. Spin angular momentum (SAM) (left), where the electric field is twisting, and orbital angular momentum (OAM) (right), where the phase does the twisting [4] (a)

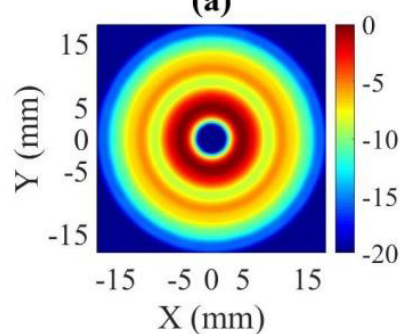

(b)

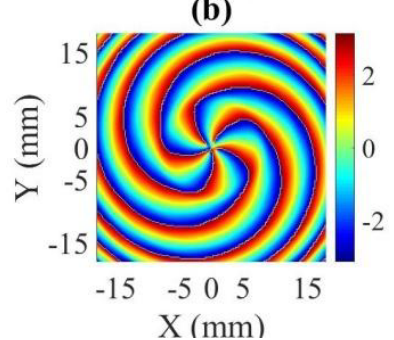

(a)

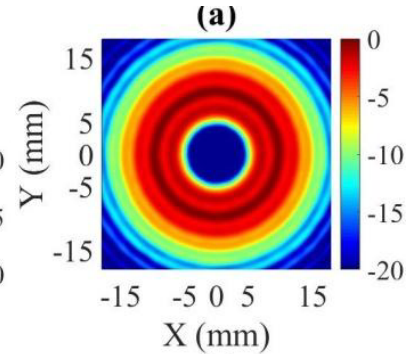

(b)

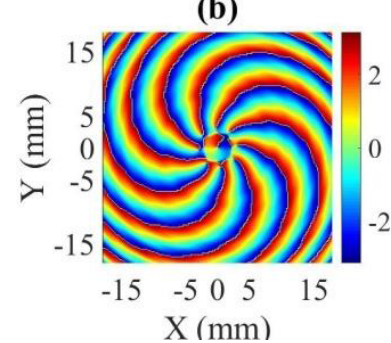

Fig. 2. Theoretical amplitude (a) and phase (b) of the timeaveraged total electric field of the co-rotating $95 \mathrm{GHz}$ modes $T E_{6,2}$ (left) and $T E_{10,1}$ (right) (in $10 \mathrm{~mm}$ distance from a $20 \mathrm{~mm}$ diameter aperture)

Figure 2 shows the analytically calculated amplitude and phase of the time-averaged total electric field of the co-rotating $95 \mathrm{GHz} T E_{6,2}$ and $T E_{10,1}$ mode, respectively. The phase patterns show $m-1$ spirals.
Several Ku/Ka-band 10-to-30-kW-class gyrotrons for technological applications operate in a low-order OAM cavity mode (e.g. $T E_{2,1}, T E_{2,2}, T E_{3,2}$, or $T E_{2,3}$ ) which also serves as axial output mode [6].

In the early 1990-ties the Research Center Karlsruhe (FZK, now KIT) performed very successful experiments with an axial output $140 \mathrm{GHz}, 0.5 \mathrm{MW} \mathrm{TE} 10,4$-mode gyrotron [7] having an electronic efficiency of approximately $33 \%$. The tube was equipped with a non-linear up-taper from the $16.22 \mathrm{~mm}$ diameter cavity to the $70 \mathrm{~mm}$ diameter output waveguide with a frequency tunable doubledisk, FC75 face cooled sapphire window. The high-power measurement of the output mode content using a $70 \mathrm{~mm}$ diameter wavenumber spectrometer [8] resulted in a purity of the co-rotating $T E_{10,4}$ output mode better than $98 \%$.

At those times, such 0.5-1.0 MW-class short-pulse gyrotrons employing asymmetric cavity modes with nonlinear up-tapers and axial output through the collector also were built by several industrial manufacturers [6], e.g.: $8 \mathrm{GHz}, T E_{5,1}$ and $110 \mathrm{GHz}, T E_{6,4}$ (THOMSON), $84 \mathrm{GHz}$, $110 \mathrm{GHz}$ and $140 \mathrm{GHz}, T E_{15,2}$ (VARIAN), $110 \mathrm{GHz}$, $T E_{12,2}$ and $T E_{22,2}$ (VARIAN), $120 \mathrm{GHz}, T E_{12,2}$ and $T E_{22,12}$ (JAERI, TOSHIBA).

\section{References}

1. Allen, L., Beijersbergen, M.W., Spreeuw, R.J.C., Woerdman, J.P. Orbital angular momentum of light and the transformation of Laguerre-Gaussian laser modes// Phys. Rev. A 1992. V. 435, P. 8185-8189.

2. Wang, J., Yang, J.-Y., Fazal, I.M., Ahmed, N., Yan, Y., Huang, H., Ren, Y., Yue, Y., Dolinar, S., Tur, M., Willner, A.E. Terabit free-space data transmission employing orbital angular momentum multiplexing // Nat. Photon. 2012. V. 6, P. 488.

3. Fokin, A.P., Denisov, G.G., Glyavin, M.Yu., Golubiatnikov, G.Yu., Lubyako, L.V., Morozkin, M.V., Movschevich, B. Z., Tsvetkov, A.I. High precision frequency stabilization of a 100 W/263 GHz continuous wave gyrotron // Proc. 18th IEEE Int. Vacuum Electronics Conference (IVEC 2017), London, UK, 2017, GII-4.

4. Sawant, A., Choe, M.S., Thumm, M., Choi, E.M. Orbital angular momentum (OAM) of rotating modes driven by electrons in electron cyclotron masers // Accepted for publication in Scientific Reports - Nature, 2017

5. Kim, S.G., Sawant, A., Lee, I., Kim, D., Choe, M., Won, J.-H., Kim, J., So, J., Jang, W., Choi, E.M. System development and performance testing of a W-band gyrotron // J. Infrared Milli Terahz Waves 2016. V. 37, No. 3. P. 209-229.

6. Thumm, M. State-of-the-art of high power gyro-devices and free electron masers // KIT Scientific Reports 2016, V. 7717, KIT Scientific Publishing, Karlsruhe, Germany

7. Piosczyk, B., Kuntze, M., Borie, E., Dammertz, G., Dumbrajs, O., Gantenbein, G., Möbius, A., Nickel, H.-U., Thumm, $M$. Development of high-power $140 \mathrm{GHz}$ gyrotrons at KfK for applications in fusion // Fusion Technology 1992, eds. C. Ferro, M. Gasparotto, H. Knoepfel, Elsevier Science Publishers B.V. 1993 P. 618-622.

8. Kasparek, W., Müller, G. The wavenumber spectrumeter - an alternative to the directional coupler for multimode analysis in oversized waveguides // Int. J. Electronics 1988 V.64 P.5-20. 